\title{
Near-Infrared Study of Iron Knots in Cassiopeia A Supernova Remnant
}

\author{
Yong-Hyun Lee ${ }^{1}$, Bon-Chul Koo ${ }^{1}$, Dae-Sik Moon ${ }^{2}$, \\ and Michael G. Burton ${ }^{3}$ \\ ${ }^{1}$ Department of Physics and Astronomy, Seoul National University, \\ 1 Gwanak-ro, Gwanak-gu, Seoul 151-742, Republic of Korea \\ email: yhlee@astro.snu.ac.kr, koo@astro.snu.ac.kr \\ ${ }^{2}$ Department of Astronomy and Astrophysics, University of Toronto, \\ 50 St. George Street, Toronto, ON M5S 3H4, Canada \\ email: moon@astro.utoronto.ca \\ ${ }^{3}$ School of Physics, University of New South Wales, Sydney, NSW 2052, Australia \\ email: m.burton@unsw.edu.au
}

\begin{abstract}
We present the results of near-infrared (NIR) imaging and spectroscopic observations of the Galactic supernova remnant Cassiopeia A (Cas A). Applying the method of Principal Component Analysis to our broadband NIR spectra, we identify a total of 61 NIR emission knots of Cas A and classify them into three groups of distinct spectral characteristics: Heliumrich, Sulfur-rich, and Iron-rich groups. The first and second groups are of the circumstellar and supernova ejecta origin, respectively. The third group, which has enhanced iron emission, is of particular interests since it shows intermediate characteristics between the former two groups. We suggest that the Iron-rich group is knots of swept-up circumstellar medium around the contact discontinuity in Cas A and/or supernova ejecta from deep layers of its progenitor star which have recently encountered a reverse shock in the remnant.
\end{abstract}

Keywords. ISM: individual (Cassiopeia A), ISM: supernova remnants, infrared: ISM

\section{Introduction and Observations}

Cas A, at the age of $\sim 340$ yrs and distance of $\sim 3.4 \mathrm{kpc}$, is one of the brightest and youngest known supernova remnants (SNRs) in our Galaxy which has provided invaluable information about the dynamical and chemical evolutions of core-collapse SNRs in an early stage. An important observational result of Cas A is discoveries of numerous emission knots scattered around the remnant in the visible wavebands: (1) fast-moving knots (FMKs) of the supernova ejecta origin abundant with nucleosynthetic metallic elements; (2) quasi-stationary flocculi (QSFs) from circumstellar material of its progenitor star; and (3) fast-moving flocculi (FMFs) (or nitrogen-knots) thought to originate from expelled photospheric layers.

In contrast to the studies in the visible wavebands, NIR observations of Cas A have been limited to spectroscopic confirmations of a few previously-identified FMKs and QSFs (Gerardy \& Fesen 2001), together with morphological studies of its [Fe II] emission, which is exceptionally bright in its southwestern rim (Rho et al. 2003). Given the importance and usefulness of NIR observations of young SNRs, especially broadband spectroscopy enclosing [Fe II] lines for investigating ejecta from deep layers of a progenitor star and shocked circumstellar material (e.g., Koo et al. 2007; Moon et al. 2009), it is important to conduct such observations for Cas $\mathrm{A}$ as we report here.

We obtained intermediate-resolution $(R \simeq 2,700)$, broadband $(0.95-2.46 \mu \mathrm{m})$ NIR longslit spectra of eight positions of bright [Fe II] $(1.64 \mu \mathrm{m})$ emission distributed around the 


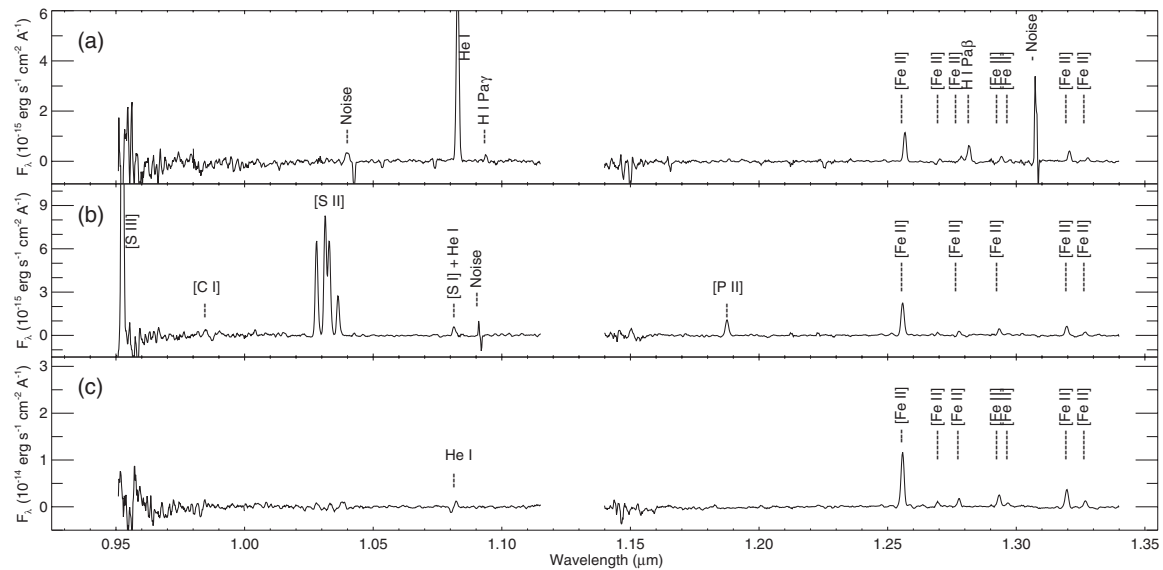

Figure 1. Sample J-band spectra of (a) Helium-rich, (b) Sulfur-rich, and (c) Iron-rich knots.

rim of Cas A with the Triplespec spectrograph of the Palomar 5-m telescope. Using the clump-finding algorithm of Williams et al. (1994), we have identified a total of 61 knots from the spectra showing 44 emission lines of $\mathrm{H}$, He, and other heavy elements in various ionized stages (see Fig. 1).

\section{Results of Principle Component Analysis}

The identified knots, 61 in total, show multivariate similarities and differences among them in their spectral characteristics such as identified lines and their intensities and velocities. In order to effectively classify the knots based on their spectral patterns, we adopt the method of Principal Component Analysis (PCA) which leads us to categorize the knots into three distinctive groups: Helium-rich, Sulfur-rich, and Iron-rich groups.

The characteristics of the knots in the Helium-rich group, which have radial velocities within $\pm 100 \mathrm{~km} \mathrm{~s}^{-1}$, are almost the same as those of the known QSFs, indicating that they are of the circumstellar nature. The knots in this group have strong emission of He I $(1.08 \mu \mathrm{m})$ and numerous [Fe II] line transitions, but without any evidence of Si, P, and S line emission. The knots in the Sulfur-rich group, on the other hand, have strong [Si VI] $(1.96 \mu \mathrm{m}),[\mathrm{P}$ II] $(1.19 \mu \mathrm{m})$, [S II] $(1.03 \mu \mathrm{m})$, [S III] $(0.95 \mu \mathrm{m})$ lines, and show increased radial velocities around $+800 \mathrm{~km} \mathrm{~s}^{-1}$ which sometimes extends to $+2,000 \mathrm{~km} \mathrm{~s}^{-1}$. These are consistent with the observed characteristics of FMKs (Gerardy \& Fesen 2001). Finally, the knots in the Iron-rich group show both the QSF-like spectral features (e.g., bright [Fe II] emission accompanied only by He I emission) and FMK-like ones (e.g., high radial velocity of several thousand $\mathrm{km} \mathrm{s}^{-1}$ ). We, therefore, suggest that they are either swept-up circumstellar material around the contact discontinuity in Cas A and/or ejecta material from deep layers, near the iron core, of the progenitor which have recently encountered a reverse shock in the SNR.

\section{References}

Gerardy, C. L. \& Fesen, R. A. 2001, AJ, 121, 2781

Koo, B.-C., Moon, D.-S., Lee, H.-G., Lee, J.-J., \& Matthews, K. 2007, ApJ, 657, 308

Moon, D.-S., Koo, B.-C., Lee, H.-G., et al. 2009, ApJL, 703, L81

Rho, J., Reynolds, S. P., Reach, W. T., et al. 2003, ApJ, 592, 299

Williams, J. P., de Geus, E. J., \& Blitz, L.1994, ApJ, 428, 693 